\title{
SOME ASPECTS OF LAMELLAR STRUCTURE IN VARIOUS GREEN LEAF PARTICLES, INDICATED BY OLEIC ACID EFFECTS
}

\section{WILLEMKE TERPSTRA}

Biophysical Research Group, Institute of Physics, State University, Utrecht (The Netherlands)

(Received October 30th, 1973)

(Revision received January 10th, 1974)

\section{SUMMARY}

(1) The influence of oleic acid on "whole chloroplasts and large fragments", isolated from spinach or endive leaves, was compared to that on Photosystem I (PS I) and PS I+II particles, probably stroma lamellae, isolated from the same leaves.

(2) Photochemical activity (Hill reaction and NADP ${ }^{+}$photoreduction) in "whole chloroplasts and large fragments" was inhibited by oleic acid; the effect was detectable at an oleic acid/chlorophyll ratio of 1-2:1. This same ratio causes a measurable change in absorption and low temperature fluorescence spectra. Photochemical activities in PS I and to a lesser extent in PS I+II particles were much less sensitive to oleic acid. The same holds for the absorbance and fluorescence of these particles.

(3) Proteins were released from thoroughly washed lamellae of the various green leaf particles by a freeze-thawing procedure. The protein pattern, obtained on disk-gel electrophoresis, differed quantitatively in the various particles, the lamellae from PS I and PS I+II particles releasing a greater proportion of high-molecular weight proteins than those of "whole chloroplasts and large fragments". Upon treatment of the lamellae with oleic acid, proteins from "whole chloroplasts and large fragments" deaggregated, whereas proteins from PS I and PS I+II lamellae were not, or much less, affected.

(4) It is concluded that oleic acid interacts with the proteins surrounding the chlorophyll in lamellae of "whole chloroplasts and large fragments". In PS I and PS I+II stroma lamellae, proteins are protected in such a way that interaction with oleic acid is prevented.

Abbreviations: DPIP, 2,6-dichlorophenolindophenol; PS, photosystem. 


\section{INTRODUCTION}

In addition to whole chloroplasts and large chloroplast fragments, homogenates of green leaves, prepared in an isotonic medium, contain small green particles with PS I or PS I+II properties [1, 2]. The small green particles are likely to be stroma lamellae $[3,4](c f$. also refs. 5, 6). In investigating chlorophyllase activity in various particles, it was found [4] that the chlorophyll $\rightarrow$ chlorophyllide conversion was increased in small particles with PS I and PS I+II properties upon addition of oleic acid at certain concentrations, whereas at the same concentrations, this conversion was inhibited in "whole chloroplasts and large fragnients". The varying effects of oleic acid may be due to its interaction with differently structured membranes ( $c f$. also refs. 7-11). It seemed worthwhile, therefore, to extend the investigations on the mode of action of oleic acid, in order to obtain some information about the structure of lamellar membranes in the various green particles.

\section{MATERIALS AND METHODS}

Spinach (Spinacia oleracea L.) and endive (Cichorium endivia L.) were obtained from the local market. Chemicals used were of reagent grade. Oleic acid, linolic acid, linolenic acid and oleic acid methyl ester were purchased from E. Merck AG, Darmstadt.

\section{Preparation of green particle fractions}

The green fraction, sedimenting at $12000 \times g$ for $20 \mathrm{~min}$ from a filtered leaf homogenate in $0.02 M$ Tris- $\mathrm{FiCl}$ buffer with $0.35 \mathrm{M}$ sucrose, contains "whole chloroplasts and large fragments" (Fraction I-III, refs. 1, 12). The supernatant of the centrifuged homogenate contains PS I particles ("Fraction 2 refs. 1, 12) and PS I+II particles ("Fraction 4", refs. 1, 12). They were separated by sucrose density gradient centrifugation. The PS I fraction contains yellow-green particles of about $0.15 \mu$, which have PS I properties. They are probably derived from stroma lamellae [3]. The PS I+II fraction contains green particles of about $0.30 \mu$, with both PS I and II properties. They are probably also stroma lamellar particles [3]. Full details of the procedure were given in refs. 1 and 12.

All particles were washed with $0.02 M$ Tris $-\mathrm{HCl}$ buffer, $\mathrm{pH}$ 8.0, containing $0.35 M$ sucrose. They were respun down at $12000 \times g$, for $20 \mathrm{~min}$ ("whole chloroplasts and large fragments") or $250000 \times \mathrm{g}$ for $1 \mathrm{~h}$ (PS I and PS I+II particles). If not mentioned otherwise, they were suspended in $0.02 M$ Tris$\mathrm{HCl}$ buffer, $\mathrm{pH} 8.0$, containing $0.35 \mathrm{M}$ sucrose.

\section{Analyses}

Absorption spectra were recorded in a Shimadzu MPS-50 L spectrophotometer. Absorption difference spectra were corrected for a "zero line", obtained by recording the apparent difference between identical particle 
suspensions, before the addition of oleic acid. Oleic acid and other long-chain molecules (linolic acid, linolenic acid, oleic acid methyl ester) were added as dilute suspensions in $0.02 \mathrm{M}$ Tris- $\mathrm{HCl}$ buffer, $\mathrm{pH} 8.0$.

Fluorescence spectra were measured at $-196^{\circ}$ with the apparatus described by Goedheer [13]. They were not corrected for photomultiplier sensitivity, but the combination of photomultiplier and monochromator was chosen in such a way that deviations in the spectrum, due to photomultiplier sensitivity, were below $10 \%$ in the $650-750 \mathrm{~nm}$ region. Particle suspensions were mixed with glycerol (final glycerol concentration $60 \%$ ) and measured in a perspex $1 \mathrm{~mm}$ cuvette placed against the window of a three-walled Dewar vessel, filled with liquid nitrogen [14].

PS II activity was measured using DPIP as Hill acceptor, whereas PS I activity was measured with $\mathrm{NADP}^{+}$as acceptor and reduced DPIP as donor (cf. ref. 1). Ferredoxin was prepared from an acetone precipitate of spinach leaves [15]. Extraction of the enzyme from the acetone precipitate and subsequent purification on DEAE cellulose columns was done according to Borchert and Wessels [16]. The absorbance ratio $A_{442 \mathrm{~nm}} / A_{274} \mathrm{~nm}$ was $>0.1$.

Reaction mixtures are given in the legends to Figs. 3 and 4 . Chlorophyll was determined using the method of Bruinsma [17].

For isolation of lamellar proteins, particles suspended in $0.01 \mathrm{M}$ Tris- $-\mathrm{HCl}$ buffer, $\mathrm{pH}$ 8.0, containing $0.35 M$ sucrose, were washed with the same buffer, for elimination of contaminating protoplasmic proteins. For further isolation of lamellae, especially from "whole chloroplasts and large fragments", an osmotic shock was produced by suspending the particles in the same Tris $-\mathrm{HCl}$ buffer without sucrose. The resulting lamellae vere washed twice; the second washing occurred for one night at about $4^{\circ}$. After this procedure, the lamellae were assumed to be free of extrinsic proteins $[18,20]$. They were spun down at $250000 \times \mathrm{g}$ (tube bottom) for $1 \mathrm{~h}$ and resuspended in $0.01 \mathrm{M}$ Tris $-\mathrm{HCl}$ buffer, $\mathrm{pH}$ 8.0. The further procedure, described below, was based on that used by Tzagoloff et al. [21] for the solubilization of mitochondrial inner membranes. In the first experiments, lamellae suspended in $0.01 \mathrm{M}$ Tris $-\mathrm{HCl}$, $\mathrm{pH} 8.0$, were sonicated $2-5$ times for $1 \mathrm{~min}$ at $800 \mathrm{kHz}$ in ice water. Later, it was found that freeze-thawing gave about equal results, as judged from protein output, whereas the chance of protein denaturation is much smaller. Freeze-thawing occurred in a thin $(0.4 \mathrm{~mm})$, stainless steel tube $(\varphi 9 \mathrm{~mm})$, by freezing 3 times for $20 \mathrm{sec}$ in liquid nitrogen and thawing 3 times for $60 \mathrm{sec}$ in running tap water. Addition of oleic acid, suspended in Tris buffer, occurred before the freeze-thawing procedure (oleic acid/chlorophyll 8:1). The resulting suspension was centrifuged at $81000 \times \mathrm{g}$ (tube bottom) for $1 \mathrm{~h}$ at about $4^{\circ}$ (cf. ref. 21 ). In the slightly yellow coloured clear supernatant, protein was determined according to Lowry et al; [22], with bovine serum albumin as a standard. Appropriate corrections were made for any colour produced by chemicals in the reaction mixture. Disk-gel electrophoresis (about $25 \gamma$ protein/gel) was performed according to Davis [23]. The gel mixture was $5 \%(\mathrm{w} / \mathrm{v})$ acrylamide, the current through each tube was $2 \mathrm{~mA}$. Gels were 
stained for proteins in Coomassie Brilliant Blue ( $0.1 \%$ solution in methanol/ acetic acid/water 1:1:8) for $4 \mathrm{~h} \mathrm{[24].} \mathrm{Destaining} \mathrm{occurred} \mathrm{in} \mathrm{7 \%} \mathrm{acetic} \mathrm{acid.}$ Gels were scanned in a Shimadzu MPS-50 L spectrophotometer at $520 \mathrm{~nm}$.

\section{RESULTS}

\section{Absorption spectra}

At "low" acid concentration (oleic acid/chlorophyll 1-8:1), the absorption difference spectra show that a decrease occurs in the absorption at $682 \mathrm{~nm}$ in the suspensions containing oleic acid, whereas a small increase is seen at about $665 \mathrm{~nm}$ (cf. also ref. 25) (Fig. 1). This effect is found with "whole chloroplasts and large fragments", as well as with PS I and PS I+II particles. However, the oleic acid/chlorophyll ratios necessary to obtain a comparable visible effect are, roughiy, 1:1 for "whole chloroplasts and large fragments", 4:1 for PS I+II particles and 8:1 for PS I particles. Apparently, "whole chloroplasts and large fragments" are more "sensitive" to oleic acid than the small stroma lamellar particles.

The same results as those obtained with oleic acid were found with linolic acid and linolenic acid, but not with oleic acid methyl ester. This indicates
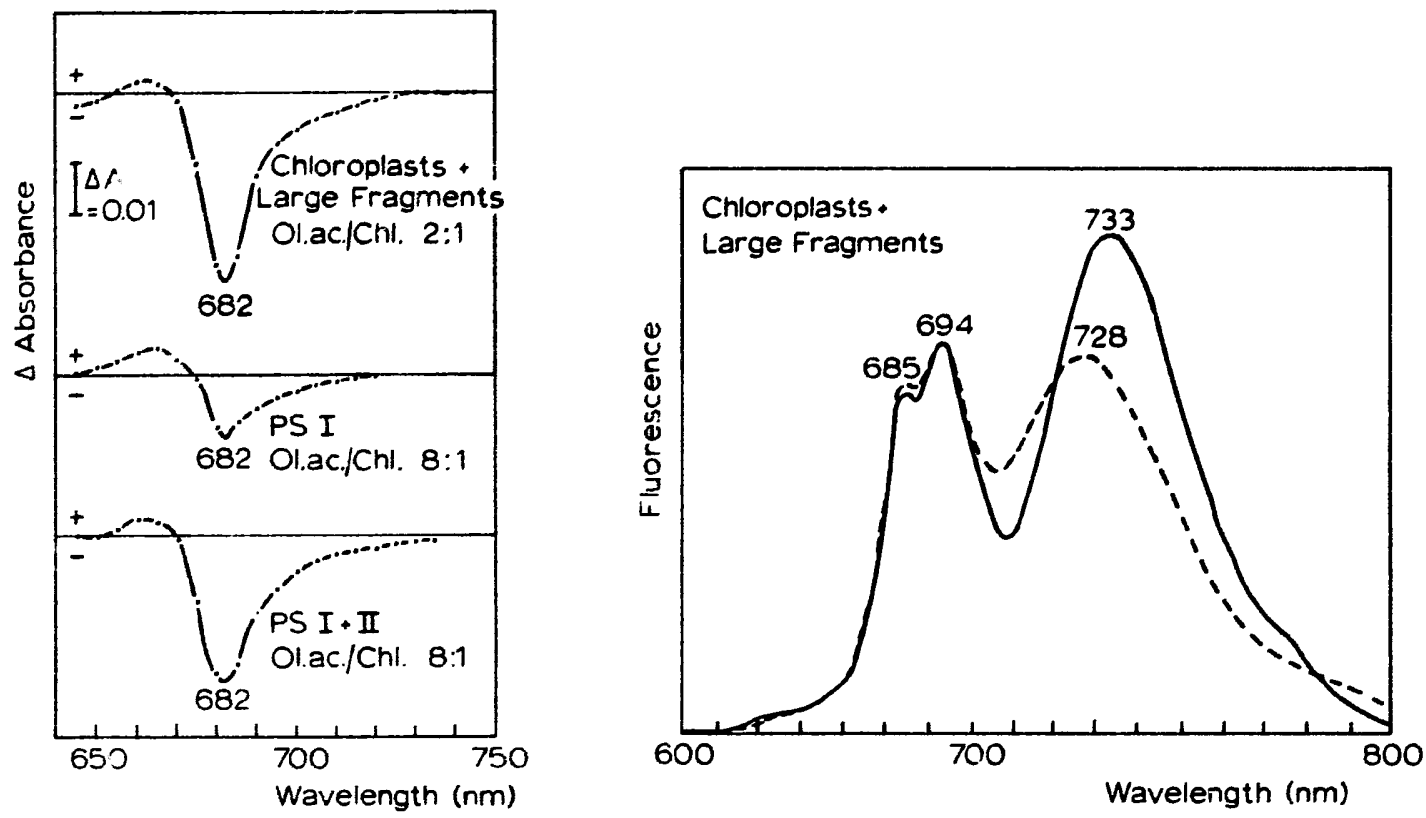

Fig. 1. Absorption difference spectra of spinach "whole chloroplasts and large fragments", PS I and PS I+II particles, with and without oleic acid. Particles suspended in $0.1 M$ phosphate buffer, pH 7.0. Chlorophyll $5 \cdot 10^{-5} M$, length of optical path $1 \mathrm{~cm}$.

Fig. 2. Effect of oleic acid (- -) at low concentrations on the fluorescence spectrum $\left(-196^{\circ}\right)$ of spinach "whole chloroplasts and large fragments". Paiticles suspended in $0.1 \mathrm{M}$ phosphate buffer, oH 7.0. Ratio of oleic acid/chlorophyll 8:1. Chlorophyll $5 \cdot 10^{-5} M$, length of optical path $1 \mathrm{~mm}$. Spectra were equalized at $694 \mathrm{~nm}$. 
that a dissociated acid group is required for the observed effects to occur.

At "high" oleic acid concentration (oleic acid/chlorophyll 25:1), absorption decreases were also found at about $495 \mathrm{~nm}$ and $440 \mathrm{~nm}$ (cf. ref. 26). The decrease at $435 \mathrm{~nm}$ indicates that the spectrum of carotenoids ( $\beta$-carotene) is affected. The changes in the spectrum are somewhat obscured by an increased light scattering in the suspensions containing oleic acid.

\section{Fluorescence spectra}

Upon addition of oleic acid, in a ratio of oleic acid/chlorophyll 8:1, to spinach "whole chloroplasts and large fragments", the $733 \mathrm{~nm}$ fluurescence naximum is increased as compared to 685 and $694 \mathrm{~nm}$ maxima, and shifted to a lower wavelength (Fig. 2). A very small effect could still be detected at a ratio of oleic acid/chlorophyll 1:1.

Even at a very high oleic acid conceintration (oleic acid/chlorophyll 570:1), only small changes occur in the 600-800 $\mathrm{nm}$ fluorescence spectrum of PS I and PS I+II particles: a slight decrease of the ratio $733 \mathrm{~nm}$ vs. 685 and $695 \mathrm{~nm}$ fluorescence is observed. In "whole chloroplasts and large fragments", at this high oleic acid concentration, the $733 \mathrm{~nm}$ fluorescence maximum has nearly disappeared ( $c f$. also refs 25,28 ). The latter experiments were done with endive. It was ascertained that no essential differences exist between spinach and endive, with reference to chlorophyll fluorescence and oleic acid effec: ; $[25,2]$.

\section{Photochemical activity}

$N A D P^{+}$photoreduction (PS I activity). With spinach "whole chloroplasts and large fragments", NADP ${ }^{+}$photoreduction is nearly completely inhibited by oleic acid at a ratio of oleic acid/chlorophyll 8:1 (Fig. 3). An effect was already observed at a ratio of oleic acid/chlorophyll 1:1 or 2:1. With PS I particles, even at a ratio of oleic acid/chlorophyll 8:1, no, or ne.urly no, inhibition was observed. The sensitivity of $\mathrm{NADP}^{+}$photoreduction in PS I+II particles is intermediary between those of "whole chlorisplasts and large fragments" and PS I particles.

Inhibition of $\mathrm{NADP}^{+}$photoreduction by oleic acid was only dependent on the ratio of oleic acid/chlorophyll and not on oleic acid concentration in the reaction mixture.

DPIP photoreduction (PS II activity). With "whole chloroplasts and large fragments", DPIP photoreduction is completely inhibited at an oleic acid/ chlorophyll ratio 8:1, whereas a small effect already occurs at a ratio of $1: 1$ or 2:1. With PS I+II particles, DPIP photoreduction is less sensitive to oleic acid (Fig. 4). PS I particles exhibit no Hill activity.

The conclusion of Krogman and Jagendorf [26], that inhibition of DPIP photoreduction by oleic acid in chloroplasts depends on the oleic acid/ chlorophyll ratio and not on oleic acid concentration, was confirmed. 




DPIP photoreduction ( $\mu$ moles/mg Chl/h)

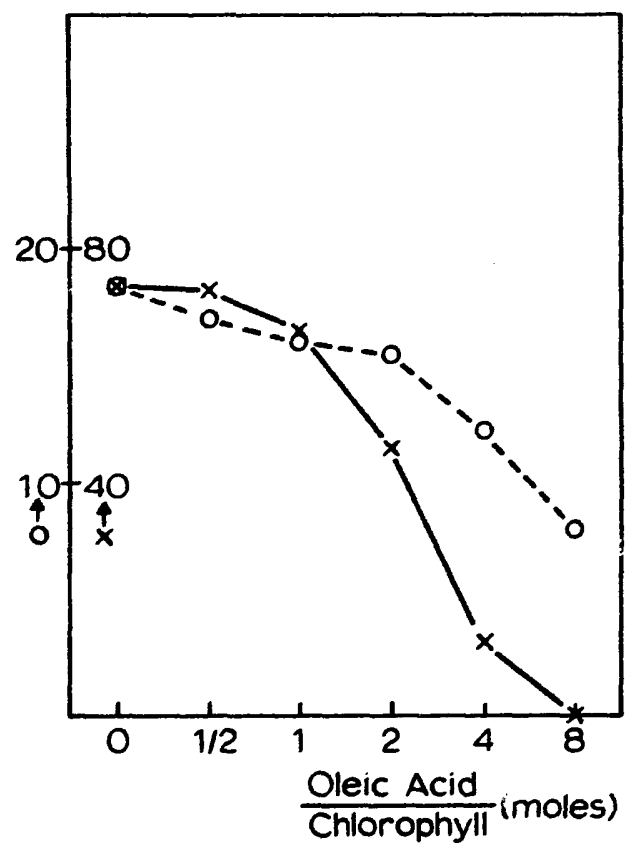

Fig. 5. Effect of oleic acid on NADP ${ }^{+}$photoreduction in spinach "whole chloroplasts and large fragments"' $(x)$, PS I $(\Lambda)$ and PS I+II ( $)$ particles. Reaction mixture: NADP ${ }^{+}$ $0.9 \mu$ moles, DPIP $0.2 \mu$ moles, sodium ascorbate $20 \mu$ mcles, excess ferredoxin and ferredoxin reductase (partially purified preparations), phosphate buffer, $\mathrm{pH}$ 7.0, $0.017 \mathrm{M}$, Tris-HCl buffer, pH 8.0, $0.05 \mathrm{M}$, chlorophyll in particles $13 \mu \mathrm{g}$, oleic acid as indicated, total volume $3.0 \mathrm{ml}$. Illumination time $4 \mathrm{~min}$ (PS I particles) or $12 \mathrm{~min}$ (PS I+Il particles and "whole chloroplasts and large fragments"). Activity was determined 1 day after preparation of the particles.

Fig. 4. Effect of oleic acid on DPIP photoreduction in spinach "whole chloroplasts and large fragments" $(x)$, and in PS I+II particles (O). Reaction mixture: DPIP $0.08 \mu$ moles, $\mathrm{KCl}$ $30 \mu$ moles, sucrose $0.25 M$, phosphate buffer, pH 7.0, $0.017 \mathrm{M}$, Tris-HCl buffer, $\mathrm{pH} 8.0$, $0.015 \mathrm{M}$, chlorophyll in particles $13 \mu \mathrm{g}$, oleic acid as indicated, total volume $3.0 \mathrm{ml}$. Illumination time $4 \mathrm{~min}$ (PS I+II particles) or $2 \mathrm{~min}$ ("whole chloroplasts and large fragments"). Activity was determined 1 day after preparation of the particles.

\section{Lamellar proteins}

The protein patterns, obtained by disk-gel electrophoresis of extracts from thoroughly washed and then freeze-thawed lamellae, differ appreciably for the varicus particles (Fig. 5). Although in all cases the same proteins appear to be present, the relative amount of high molecular weight proteins, at only a small distance from the origin in the running gel, is gieatest in stroma lamellar fractions. On the other hand, the very clear band, found at a relative distance of about 0.69 in extracts from "whole chloroplasts and large fragments", is nearly absent in those of PS I particles. 

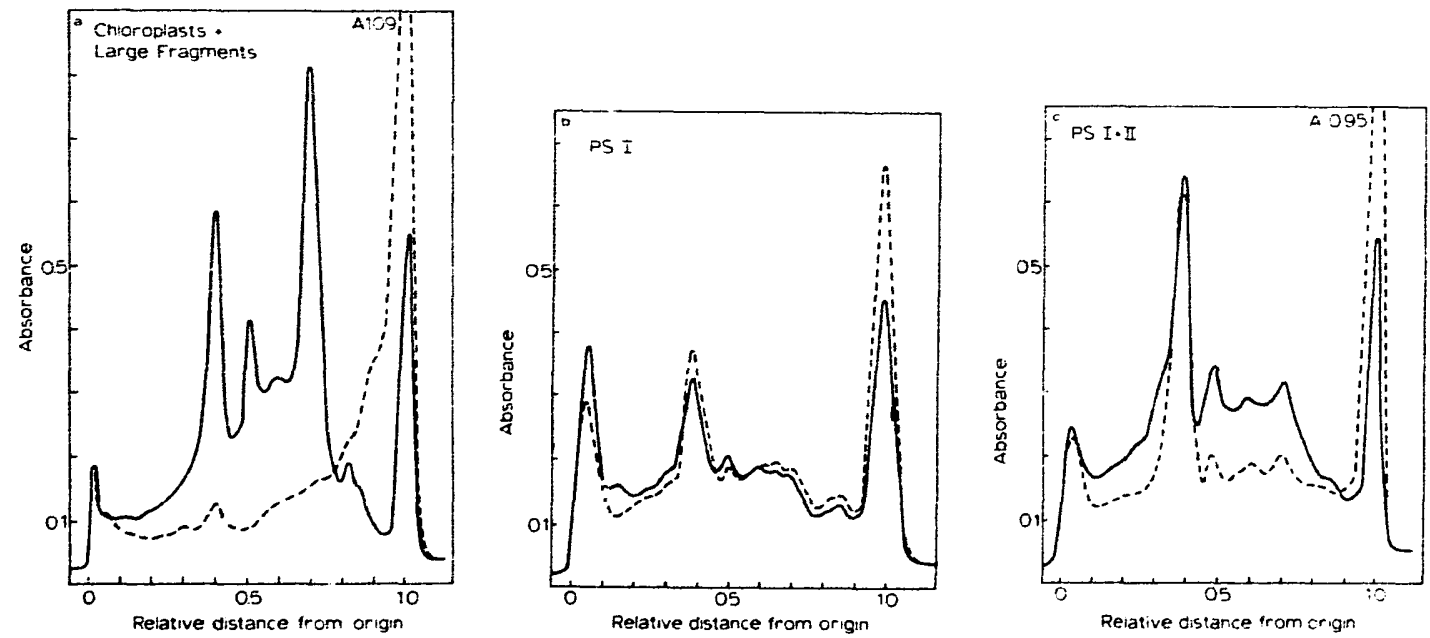

Fig. 5. Disk-gel electrophoresis protein patterns of lameilar extracts frcm spinach "whole chloroplasts and large fragments", PS I and PS I+II particles before $(-)$ and after treatment of the green particles with oleic acid (- - ). Ratio of oleic acid/chlorophyll 8:1.

Even though equal amounts of protein were applied to each gel, the total amount of protein, present in the running gel, is greater with "whole chloroplasts and large fragments" than with PS I, and, to a lesser extent, PS I+II particles. Apparently, PS I and PS I+Il particles had released a relatively high proportion of high-molecular weight proteins which were retained in the sample gel.

Treatment of the green particles with oleic acid (oleic acid/chlorophyll 8:1) does not significantly influence, quantitatively, the amount of protein that is released by the lamellae during the freeze-thawing procedure, However, upon disk-gel electrophoresis, a marked shift of high molecular weight to lower molecular weight proteins occurs in extracts of "whole chloroplasts and large fragments" and to a lesser degree in those of PS I+II particles, whereas PS I particle extracts only show very small or no changes (Fig. 5).

It may be noted that some proteins, e.g. those at relative distances of about 0.39 and 0.50 , are sensitive to oleic acid treatment in "whole chloroplasts and large fragments", whereas they are insensitive to the same treatment in PS I and PS I+II particles.

For investigation of oleic acid influence on lamellar proteins, it was necessary to obtain these proteins without utilizing detergents (cf. ref. 27), as the latter are very likely to influence oleic acid-protein interaction. Although, with our procedure, solubilization of intrinsic proteins from lamellar membranes is certainly incomplete, the results show that at least a fair number of proteins are released. 
Measurable effects of oleic acid on absorption and fluorescence spectra of spinach "whole chloroplasts and large fragments", as well as on their photochemical activity, occurred at the same range of oleic acid/chlorophyll ratios (1-2:1). Although these results may depend on the sensitivity of its measurements, the conclusion that the mentioned effects have the same origin seems plausible. "Whole chloroplasts and large fragments" contain a relatively large fraction of granar lameilae, as a large part of the stroma lamellae (PS I and PS I+II particles) has been eliminated by cifferential centrifugation of the spinach leaí homogenate (cf. METHODS). As NADP ${ }^{+}$photoreduction (PS I activity), as well as DPIP photoreduction (PS II activity), were inhibited by oleic acid to the same extent, it is concluded that oleic acid affects both granar phot usystems in the same way. This conclusion is not in agreement with that of Cohen et al. [28]. These authors; as well as Siegenthaler [29], report that, with spinach chloroplast preparations, the electron flow activity of PS I is affected by fatty acids at a higher concentration than that necessary for affecting PS II (cf. also ref. 39). The difference with the results of the present study may be due to the presence, in the mentioned authors' chloroplast preparations, of a larger quantity of PS I (and PS I+II) stroma lamellae, which were found to be very sensitive to oleic acid (see below). Besides, in these experiments with a lipophylic molecule, lipophilicity of electron donors and acceptors and other reagents used, might be of importance [31].

With PS I and PS I+II stroma lamellar particles, the oleic acid concentration needed to obtain a measurable change in the absorption spectrum, is about 8 (PS I) or 4 (PS I+II) times that which is found to be required for an equal absorption change in "whole chloroplasts and large fragments". For a measurable inhibition of photochemical activity in stroma lamellar particles, the same 8-4 times higher oleic acid quantity than that needed for such an inhibition in "whole chloroplasts and large fragments" is required. This seems to be a strong argument for the suggestion, put forward in the first paragraph of this section, that the effect of oleic acid on absorption spectra, as well as that on the photochemical activity of the various particles, has the same molecular origin.

Goedheer $[13,32]$ ascribes the presence of a high long-wavelength $(733 \mathrm{~nm})$ fluorescence maximum in low temperature spectra of higher plant chloroplasts, to an increased energy transfer to the fluorescing long-wave chlorophyll form upon cooling. Oleic acid might then interfere with energy transfer in "whole chloroplasts and large fragments", but not, or to a lesser degree, in PS I and PS I+II particles.

From the results mentioned above, it is concluded that oleic acid affects the immediate environment of chlorophyll, from both photosystems, in membranes of "whole chloroplasts and large fragments", but that the environment of chlorophyll molecules in stroma lamellae is not, or much less, influenced. 
Brody and Nathansor: 10$]$ reported the influence of fatty acids on circular dichroism spectra of chiorophy $11-$ protein complexes, prepared according to Thornber [33], and of chlor splasts, digitonin-isolated particles containing PS I and particles containing PS I and II. The changes induced by fatty acids were interpreted to be due to "deaggregation of chlorophyll, resulting from conformational changes in protein".

In view of our ow: conclusions, and those of Brody and Nathanson [10], it seemed worthwhile to investigate if any influence of oleic acid on membrane proteins could be detected. Experimental results showed that such an influence was indeed found (Fig. 5). The proteins, released upon freeze-thawing, are qualitatively the same in all kinds of particles. Oleic arid treatment of "whole chloroplasts and large fragments" induces a shift to low molecular weight proteins; this suggests that oleic acid causes a deaggregation of proteins. The deaggregating effect of oleic acid is much more pronounced in "whole chloroplasts and large fragments" than in PS I+II particles, whereas in PS I particles the effect is nearly absent. This led us to assume that, in stroma lamellar particles, protein aggregates are protected in one way or anotner. The lipids in the membrane may tentatively be suggested to play a role as protective agents (c $f$. ref. 4 ).

To sum up, it is concluded that oleic acid interacts with the proteins surrounding the chlorophyll in lamellae of "whole chloroplasts and large fragments". In PS I and PS I+II stroma lamellae, preteins are protected in such a way that interaction with oleic acids is more or less prevented.

\section{ACKNOWLEDGEMENT}

It is a pleasure to acknowledge expert technical assistance of Mrs. W. Baas.

\section{REFERENCES}

1 W. Terpstra, Biochim. Biophys. Acta, 216 (1970) 179.

2 W. Terpstra, in G. Forti, M. Avron and A. Melandri (Eds.), Proc. IInd Incern. Congr. on Photosynthesis, Stresa, 1971, pp. 1545.

3 W. Terpstra, Z. Pflanzenphysiol., 67 (1972) 255.

4 W. Terpstra, Z. Pflanzenphysiol., 7 (1974) in the press.

5 P.V. Sane, D.J. Goodchild and R.B. Park, Biochim. Biophys. Acta, 216 (1970) 162.

6 R.B. Park and P.V. Sane, Ann. Rev. Plant Physiol., 22 (1971) 395.

7 Y.G. Molotkovsky and I.M. Zhestkova, Biochim. Biophys. Acta, 112 (1966) 170.

8 T.A. Pedersen, M. Kirk and J.A. Bassham, Biochim. Biophys. Acta, 112 (1966) 189.

9 T.H. Ji and A.A. Benson, Biochim. Biophys. Acta, 150 (1968) 686.

10 M. Brody and B. Nathanson, Biophys. J., 12 (1972) 774.

11 P.A. Siegenthaler, Biochim. Biophys. Acta, 275 (1972) 182.

12 W. Terpstra and A.C.M. Weijman, Planta, 108 (1972) 319.

13 J.C. Goedheer, Biochim. Biophys. Acta, 88 (1964) 304.

14 C. Bril, D.J. van der Horst, S.R. Poort and J.B. Thomas, Biochim. Biophys. Acta, 172 (1969) \&45.

15 A. San Piétro and H.M. Lang, J. Biol. Chem., 231 (1958) 211.

16 M.T. Borchert and J.S.C. Wessels, Biochim. Biophys. Acta, 197 (1970) 78. 
17 J. Bruinsma, Biochim. Biophys. Acta, 52 (1961) 576.

18 R.A. Capaldi and D.E. Green, FEBS Letters, 25 (1972) 205.

19 G. Vanderkooi, Ann. N.Y. Acad. Sci., 195 (1972) 6.

20 S.J. Singer and G.L. Nicholson, Science, 175 (1972) 720.

21 A. Tzagoloff, D.G. McConnell and D.H. McLennan, J. Biol. Chem., 243 (1968) 4117.

22 O.H. Lowry, N.J. Rosebrough, A.L. Farr and R.J. Randall, J. Biol. Chem., 192 (1951) 265.

23 B.J. Davis, Ann. N.Y. Acad. Sci., 121 (1964) 404.

24 J. Bennett and K.J. Scott, Analyt. Biochem., 43 (1971) 173.

25 B. Nathanson and M. Brody, Photochem. Photobiol., 12 (1970) 469.

26 D.W. Krogmann and A.T. Jagendorf, Arch. Biochem. Biophys., 80 (1959) 421.

27 F.A. McEvoy and W.S. Lynn, J. Biol. Chem., 248 (1973) 4568.

28 W.S. Cohen, B. Nathanson, J.E. White and M. Brody, Arch. Biochem. Biophys., 135 (1969) 21.

29 P.A. Siegenthaler, Biochim. Biophys. Acta, 305 (1973) 153.

30 S. Katoh and A. San Pietro, Arch. Biochem. Biophys., 128 (1968) 378.

31 G. Hauska, A. Trebst and W. Draber, Biochim. Biophys. Acta, 305 (1973) 632.

32 J.C. Goedheer, Ann. Rev. Plant Physiol., 23 (1972) 87.

33 J.P. Thornber, Biochim. Biophys. Acta, 172 (1969) 230. 